\title{
Wisdom and fluid intelligence are dissociable in healthy older adults
}

\author{
Cutter A. Lindbergh, ${ }^{1}$ Heather Romero-Kornblum, ${ }^{1}$ Sophia Weiner-Light, \\ J. Clayton Young, ${ }^{1}$ Corrina Fonseca, ${ }^{1}$ Michelle You, ${ }^{1}$ Amy Wolf, ${ }^{1}$ Adam M. Staffaroni, ${ }^{1}$ \\ Rebecca Daly, ${ }^{2,3}$ Dilip V. Jeste, 2,3,4 Joel H. Kramer, ${ }^{1}$ Winston Chiong, ${ }^{1}$ \\ and the Hillblom Aging Network \\ ${ }^{1}$ Memory and Aging Center, Department of Neurology, University of California San Francisco, San Francisco, CA, USA \\ ${ }^{2}$ Department of Psychiatry, University of California San Diego, La folla, CA, USA \\ ${ }^{3}$ Sam and Rose Stein Institute for Research on Aging, University of California San Diego, La folla, CA, USA \\ ${ }^{4}$ Department of Neurosciences, University of California San Diego, La folla, CA, USA
}

Objectives: The relationship between wisdom and fluid intelligence (Gf) is poorly understood, particularly in older adults. We empirically tested the magnitude of the correlation between wisdom and Gf to help determine the extent of overlap between these two constructs.

Design: Cross-sectional study with preregistered hypotheses and well-powered analytic plan (https://osf.io/ h3pjx).

Setting: Memory and Aging Center at the University of California San Francisco, located in the USA.

Participants: 141 healthy older adults (mean age $=76$ years; $56 \%$ female).

Measurements: Wisdom was quantified using a well-validated self-report-based scale (San Diego Wisdom Scale or SD-WISE). Gf was assessed via composite measures of processing speed (Gf-PS) and executive functioning (Gf-EF). The relationships of SD-WISE scores to Gf-PS and Gf-EF were tested in bivariate correlational analyses and multiple regression models adjusted for demographics (age, sex, and education). Exploratory analyses evaluated the relationships between SD-WISE and age, episodic memory performance, and dorsolateral and ventromedial prefrontal cortical volumes on magnetic resonance imaging.

Results: Wisdom showed a small, positive association with Gf-EF ( $\mathrm{r}=0.181$ [95\% CI 0.016, 0.336], $\mathrm{p}=.031$ ), which was reduced to nonsignificance upon controlling for demographics, and no association with Gf-PS $(\mathrm{r}=0.019$ [95\% CI $-0.179,0.216], \mathrm{p}=.854)$. Wisdom demonstrated a small, negative correlation with age $(\mathrm{r}=-0.197$ [95\% CI $-0.351,-0.033], \mathrm{p}=.019)$, but was not significantly related to episodic memory or prefrontal volumes.

Conclusions: Our findings indicate that most of the variance in wisdom ( $>95 \%)$ is unaccounted for by Gf. The independence of wisdom from cognitive functions that reliably show age-associated declines suggests that it may hold unique potential to bolster decision-making, interpersonal functioning, and other everyday activities in older adults.

Key words: aging, cognition, executive functioning, neuroimaging, processing speed

\section{Introduction}

Wisdom is a complex, multidimensional personality trait, involving cognitive, social, and emotional processes, which has been referenced in ancient texts for

Correspondence should be addressed to: Dr. Cutter A. Lindbergh, University of California San Francisco, Memory and Aging Center Box 1207, 675 Nelson Rising Lane, Suite 190, San Francisco, CA 94143. Phone: 1-406-493-7072. Email: Cutter. Lindbergh@ucsf.edu Received 30 Dec 2020; revision requested 06 Feb 2021; revised version received 19 Feb 2021; accepted 30 Mar 2021. First published online 10 May 2021. thousands of years (Jeste and Lee, 2019; Jeste and Vahia, 2008). However, it was not until the 1970s that the construct of wisdom began to be systematically studied within the context of empirical research (Thomas et al., 2019). Since then, wisdom has been shown to be associated with a range of positive health outcomes, including physical health, mental health, happiness, life satisfaction, social connectedness, and psychological resilience (Ardelt and Ferrari, 2019; Auer-Spath and Gluck, 2019; Jeste et al., 2020; Lee et al., 2019; Webster et al., 2014). It 
is among a relatively small number of characteristics that is popularly thought to increase (as opposed to decline) in older ages and may help explain why, from an evolutionary perspective, it is advantageous for members of certain species to live well beyond the age of reproductive capability (Jeste and Lee, 2019).

There is considerable and long-standing debate regarding the nature of the relationship between wisdom and the potentially overlapping construct of fluid intelligence (Gf) (Cattell, 1943). Gf, or "intelligence-as-process," involves the ability to efficiently generate, transform, manipulate, and reason with novel information to accomplish goals and solve problems (McGrew, 2009). It is often measured by cognitive tests of information processing speed (Gf-PS) and executive functioning (Gf-EF) (Diamond, 2013; Kievit et al., 2016; Sheppard and Vernon, 2008; Van Aken et al., 2015). Clarifying the empirical delineation of wisdom and Gf as constructs is not only important from a theoretical perspective but carries important practical implications as well. In particular, Gf has been found to reliably decline in later life (Salthouse, 2004) and is the single greatest cognitive predictor of functional ability among older adults (Cahn-Weiner et al., 2007). To the extent that wisdom is dissociable from Gf, wisdom may hold potential to compensate for age-related cognitive declines by bolstering decision-making, interpersonal skills, and other aspects of everyday functioning.

According to the "complementary" theory, wisdom and Gf may share some commonalities, but the degree of overlap is small, and the two constructs are by and large distinct (Jeste et al., 2010). At most, Gf may help facilitate wisdom, but possessing intelligence is considered necessary but insufficient for being wise (Jeste et al., 2020). Proponents of the complementary view point to the multidimensional nature of wisdom, which involves social and emotional processes (e.g. empathy, compassion, affect regulation, self-reflection, social advising) in addition to cognitive skills, and thus argue that wisdom is much broader than Gf (Jeste and Lee, 2019; Thomas et al., 2019). Multidimensional measures of wisdom, such as the Three-Dimensional Wisdom Scale (3D-WS), have increasingly emerged over the last two decades to assess affective and reflective components of wisdom in addition to cognitive aspects (Ardelt, 2003). Importantly, such scales have demonstrated strong psychometric properties (validity and reliability), lending credence to a multidimensional approach to the wisdom construct (Ardelt, 2003).

The complementary view is further supported by findings that core aspects of Gf, such as Gf-EF and Gf-PS, consistently decline in later life (Diamond,
2013), whereas empirical studies of the relationship between age and wisdom have yielded inconsistent results. Although some studies have found negative age-wisdom correlations (Mickler and Staudinger, 2008; Thomas et al., 2019), others have found positive correlations (Grossmann et al., 2010; Happé et al., 1998; Worthy et al., 2011), no correlation (Smith and Baltes, 1990; Webster, 2007), or curvilinear effects (Ardelt et al., 2018; Thomas et al., 2017; Webster et al., 2014). The observation that Gf and wisdom may show distinct trajectories in aging argues against their conceptualization as a unified construct.

In contrast to the complementary theory, the "interrelated" view posits that Gf is a central component of wisdom, with some theorists defining wisdom as the application of intelligence to achieve goals and uphold values (Sternberg, 2005). This stems in part from early empirical investigations of wisdom by Baltes and colleagues, who argued that wisdom is characterized by exceptionally high levels of intelligence and therefore, is possessed by very few people (Baltes and Smith, 1990). Proponents of the "interrelated" view emphasize the commonalities, rather than the differences, between wisdom and Gf: both entail reasoning, decisionmaking processes, insight, and self-regulation skills. Indeed, traits used to describe people who are "wise" tend to be highly correlated with traits used to describe people who are "intelligent" (Sternberg, 1985). There is also overlap in the putative neuroanatomy of Gf and wisdom, with both thought to rely heavily on prefrontal brain networks (Jung and Haier, 2007; Meeks and Jeste, 2009). Empirical support for the "interrelated" view is further derived from developmental studies suggesting that wisdomlike behavior and Gf may increase in tandem during adolescence, presumably paralleling the maturation of self-reflection and other executive skills (Luna et al., 2010).

A paucity of research has empirically tested the relationship between wisdom and cognitive functioning, let alone cognitive skills specific to Gf, in older adults. Jeste and colleagues (2019) demonstrated that wisdom was positively associated with a global measure of cognitive and functional status in a sample of senior housing community residents. In addition, among middle-aged patients with schizophrenia, wisdom was positively related to cognitive performance, particularly on measures of Gf-PS (Van Patten et al., 2019). However, a more detailed understanding of the association between wisdom and specific aspects of cognitive function, especially as related to Gf, is lacking in older adults. One potential confounder is of particular importance in this population. Alzheimer's disease and other neurodegenerative conditions may have a long preclinical stage that often includes unrecognized 
cognitive decline (Sperling et al., 2011). Preclinical or otherwise undiagnosed neurodegenerative disease is known to diminish Gf and can be predicted to reduce wisdom, so failure to control for this potential confounder could induce spurious correlations between $\mathrm{Gf}$ and wisdom.

The preregistered primary aim of the proposed study was to further our understanding of the relationship between wisdom and Gf in a stringently characterized cohort of healthy older adults. Specifically, we aimed to empirically test the magnitude of the association between wisdom and Gf using an analytic plan with sufficient statistical power to detect at least a moderately sized correlation between these two constructs.

Secondary aims were to evaluate the relationships of wisdom with age, episodic memory performance (given the centrality of this cognitive function to aging and age-related neurodegenerative diseases), and brain structural volumes with a focus on prefrontal cortical regions (dorsolateral and ventromedial) that have been posited to subserve wisdom in the literature (Meeks and Jeste, 2009). Due to inconsistencies in prior findings and a generally limited research base, our secondary aims are exploratory and without specific a priori hypotheses.

\section{Methods}

The hypotheses and analytic plan for this study were preregistered using the Open Science Framework (https://osf.io/h3pjx). De-identified data (doi:10 .17605/OSF.IO/EFJNR) for our primary analyses are publicly available within the Open Science Network repository (https://mfr.osf.io/render?url= https://osf.io/dg $8 \mathrm{mk} /$ ?direct $\% 26$ mode $=$ render $\%$ 26 action $=$ download $\% 26$ mode $=$ render). The sample was drawn from a larger cohort of communitydwelling older adults enrolled in the Hillblom Aging Network (>400 active participants) - a longitudinal study of healthy brain aging at the University of California San Francisco (UCSF) Memory and Aging Center. Recruitment for the Hillblom Aging Network began in 2000 and has primarily involved flyers, newspaper advertisements, and community outreach events in the Bay Area. Participants in this cohort are verified as neurologically normal based on a multidisciplinary assessment including a neurological examination, in-person neuropsychological testing, and an informant interview. As part of the Hillblom Aging Network, participants complete online web-based tasks in addition to in-person neuropsychological testing and neuroimaging.

Inclusionary criteria for the current study were clinically normal per an informant-obtained Clinical Dementia Rating (CDR global score $=0$ ) and willingness to complete an online version of the San Diego Wisdom Scale (SD-WISE) that was distributed via email (described below). Because the online web-based tasks are sent to the Hillblom Aging Network asynchronously with the in-person measures, participants were required to have CDR, cognitive, and neuroimaging data within 2 years of completion of the SD-WISE in order to be included within our analyses.

The Hillblom Aging Network protocol was reviewed and approved by the UCSF Committee on Human Research. This study was conducted in full compliance with the ethical principles set forth by the Declaration of Helsinki. All participants provided written informed consent.

\section{SD-WISE}

Participants were emailed a link inviting them to participate in an online version of the SD-WISE that was programmed in Qualtrics. The SD-WISE is a 24-item self-report-based scale covering the domains of social advising, emotional regulation, pro-social behaviors, self-reflection, acceptance of divergent perspectives, and decisiveness (Thomas et al., 2019). Examples of individual items include the following: (1) "It is important that I understand the reasons for my actions"; (2) "I often don't know what to tell people when they come to me for advice"; and (3) "I enjoy being exposed to diverse viewpoints". Response options for each item are: "strongly disagree," "disagree," "neutral," "agree", or "strongly agree." The total score reflects an average of the responses to individual items, taking into account reverse-coded items, with higher scores corresponding to higher levels of wisdom (score range $=1-5$ ). The SD-WISE is firmly grounded in empirical data and theory, with item content carefully selected according to relevant ancient texts and modern scientific literature (Bangen et al., 2013; Jeste and Vahia, 2008) as well as expert consensus using the Delphi Method (Jeste et al., 2010). The SD-WISE has good-to-excellent psychometric properties, including convergent and discriminant validity, and its overall structure has been confirmed using factor analyses (Thomas et al., 2019). With respect to reliability, omega $(\omega)$, omega hierarchical $\left(\omega_{\mathrm{H}}\right)$, and internal consistency coefficient alpha $(\alpha)$ have been shown to be $0.93,0.80$, and 0.71 , respectively, which compares favorably to other widely used wisdom scales in the literature (Thomas et al., 2019).

\section{Cognitive composite measures}

Gf was quantified using sample-based composite measures of executive functioning (Gf-EF) and processing speed (Gf-PS), as described in detail in 
prior publications (e.g. Lindbergh et al., 2019; Staffaroni et al., 2018). We elected to use composite scores to capture Gf given superior psychometric properties, including reliability, as compared with individual test scores, particularly in aging populations (e.g. Jonaitis et al., 2019). Briefly, the Gf-EF composite comprises Stroop interference, modified trail making test, digit span backward, phonemic fluency, and design fluency (Delis et al., 2001). The Gf-PS composite is derived from six computerized, visually mediated, speeded tasks, including Length Judgment, Visual Search, Distance Judgment, Abstract Matching 1, Abstract Matching 2, and Shape Judgment (Hale and Myerson, 1996; Kerchner et al., 2012). Scores are normalized against healthy young adults as detailed by Kerchner and colleagues (2012).

For exploratory analyses, episodic memory was quantified using a sample-based composite measure of Benson Figure delayed recall (Kramer et al., 2003) and performance on the California Verbal Learning Test, second edition (immediate recall total, delayed recall total, and recognition discriminability; Delis et al., 2000).

Higher scores correspond to better performance for the Gf-EF and episodic memory composites. By contrast, lower scores indicate better performance (faster reaction times) on the Gf-PS composite.

\section{Brain structural measures}

A subset of participants underwent T1-weighted magnetization prepared rapid gradient-echo (MPRAGE) magnetic resonance imaging (MRI) on a 3.0 Tesla Siemens Prisma Fit scanner. The scans were acquired sagittally using the following parameters: repetition time $(\mathrm{TR})=2300 \mathrm{~ms}$, inversion time $(\mathrm{TI})=900 \mathrm{~ms}$, echo time $(\mathrm{TE})=2.9 \mathrm{~ms}$, flip angle $=9^{\circ}$, field-of-view $(\mathrm{FOV})=240 \times 256 \mathrm{~mm}$ with $1 \times 1 \mathrm{~mm}$ in-plane resolution and $1 \mathrm{~mm}$ slice thickness. Image processing included correction of magnetic field bias via the N3 algorithm (Sled et al., 1998). Tissue segmentation was achieved using SPM12's unified segmentation procedure (Friston et al., 2011), and each participant's gray matter segmentation was warped using DARTEL (Diffeomorphic Anatomical Registration using Exponentiated Lie algebra) to create a study-specific template (Ashburner, 2007). Each participant's native space gray matter segmentation was normalized and modulated, via nonlinear and rigid-body transformations, to study-specific template space. A Gaussian kernel of 4-mm full width half maximum was applied for smoothing of images. Transformations (linear and nonlinear) between DARTEL's space and International Consortium for Brain Mapping (ICBM) space were conducted to enable statistical comparisons
(Mazziotta et al., 1995). Finally, brain volumes of interest were quantified by translating a standard parcellation atlas (Desikan et al., 2006) into ICBM space and summing the gray matter within each identified region. Dorsolateral prefrontal cortex (dIPFC) consisted of bilateral caudal and rostral middle frontal gyri (Sallet et al., 2013), and ventromedial prefrontal cortex (vmPFC) consisted of bilateral medial orbitofrontal regions (Delgado et al., 2016).

\section{Statistical analyses}

For primary aim analyses, bivariate correlation coefficients (Pearson's $r$ ) were calculated to evaluate the magnitude of the relationship between wisdom (SDWISE scores) and Gf (Gf-EF and Gf-PS composite scores). In follow-up exploratory analyses, these relationships were also tested in multiple regression models covarying for age, sex, and educational attainment (in years). The a priori rationale for performing the analyses with and without demographic adjustment is that, on the one hand, the interrelated and complementary models posit that the constructs of wisdom and Gf are either related or not, regardless of demographic factors such as age, sex, or education. On the other hand, the exploratory multiple regression analysis helps inform whether core demographic variables may influence the relationship between wisdom and Gf, particularly given the paucity of empirical studies on this topic to date.

For our secondary aims, the relationship between wisdom and episodic memory composite scores was similarly investigated in both bivariate correlational analyses (Pearson's $r$ ) and multiple regression models with demographic (age, sex, and education) adjustment. The association between wisdom and brain volume regions-of-interest (ROIs; dIPFC and vmPFC) was evaluated in multiple regression analyses controlling for total intracranial volume (TIV), with and without demographic covariates in the model. Finally, bivariate correlational analyses and multiple regression models adjusting for sex and education were performed to test the relationship between age and wisdom. Based on prior literature suggesting the possibility of curvilinear associations (Ardelt et al., 2018; Thomas et al., 2017; Webster et al., 2014), the relationship between age and wisdom was probed in both linear and quadratic regression models.

In addition to our preplanned ROI-based analysis involving dIPFC and vmPFC, we performed an exploratory whole-brain voxel-based morphometry (VBM) analysis to help advance knowledge of the neuroanatomy of wisdom in older adults, given the lack of prior studies on this topic. The VBM analysis was performed in SPM using standard settings 
recommended by the developer (Friston et al., 2011). In parallel with the ROI-based analysis, the VBM analysis evaluated the association between wisdom (SD-WISE scores) and TIV-adjusted gray matter volumes with and without inclusion of demographic covariates (age, sex, and education). For statistical thresholding, the model implemented a voxelwise $\mathrm{p}<.005$ as well as a cluster size $\mathrm{p}<.05$ based on a Monte Carlo simulation with 1,000 permutations.

\section{Power analysis}

A power analysis was conducted to determine the minimum sample size necessary to reject the null hypothesis $(\rho=0)$, if it were in fact false, for our primary aim analyses evaluating the relationship between wisdom and Gf. The power analysis revealed that at least 84 participants would be required to detect a medium-sized effect $(\rho=0.30)$ with power $(1-\beta)$ of 0.80 and alpha $(\alpha)$ set to .05 (two-tailed). The available sample size for our primary analyses surpassed this threshold (see Results section, below).

\section{Results}

In total, 217 participants within the Hillblom Aging Network completed the SD-WISE. Of these 217 participants, 141 had a global CDR score of 0 and Gf-EF data within 2 years of completion of the SDWISE. Gf-PS data were available for 99 of these participants. Accordingly, for our primary aim analyses, the achieved power was 0.86 for Gf-PS $(n=99)$ and 0.95 for Gf-EF $(\mathrm{n}=141)$ to detect a mediumsized effect $(\rho=0.30)$ with $\alpha$ set to .05 (two-tailed). For our exploratory analyses, 141 participants had episodic memory data and 82 had structural MRI data within the 2-year window.

Descriptive statistics for the overall sample of eligible participants $(\mathrm{N}=141)$ are provided in Table 1 . The study sample was highly educated $(>17$ years on average) and predominantly Caucasian (92\%) with a mean age of 76 years. Measures of central tendency (e.g. mean) and dispersion (e.g. standard deviation) on the SD-WISE were comparable to those reported in other older adult cohorts, supporting its reliability (Jeste et al., 2019). Consistent with prior findings (Thomas et al., 2019), males and females demonstrated similar levels of wisdom $(t=-0.108$, $\mathrm{p}=.914$ ), and educational attainment and wisdom were not significantly correlated $(\mathrm{r}=.124, \mathrm{p}=.145)$.

\section{Gf and wisdom}

Wisdom demonstrated a positive and small but statistically significant association with Gf-EF
Table 1. Descriptive Statistics

\begin{tabular}{|c|c|c|}
\hline & MEAN (SD) OR \% & RANGE \\
\hline Age (years) & $76.66(7.57)$ & $51.84,92.27$ \\
\hline Sex (\% female $)$ & $56 \%$ & $\mathrm{n} / \mathrm{a}$ \\
\hline Education (years) ${ }^{\mathrm{a}}$ & $17.74(1.99)$ & $12.00,20.00$ \\
\hline Race $(\% \text { White })^{\mathrm{b}}$ & $92 \%$ & $\mathrm{n} / \mathrm{a}$ \\
\hline $\begin{array}{l}\text { SD-WISE (total } \\
\text { score) }\end{array}$ & $4.08(0.36)$ & $3.13,4.88$ \\
\hline Gf-EF (z-score) & $0.31(0.67)$ & $-1.77,1.91$ \\
\hline Gf-PS (z-score) ${ }^{c}$ & $2.54(1.61)$ & $-0.12,7.65$ \\
\hline $\begin{array}{l}\text { Episodic memory } \\
\quad(\mathrm{z} \text {-score) }\end{array}$ & $0.06(0.81)$ & $-2.38,1.48$ \\
\hline
\end{tabular}

Note. Descriptive statistics are presented as mean (standard deviation) with ranges (minimum, maximum) or percentages for participants who met inclusionary criteria $(\mathrm{N}=141)$.

$\mathrm{SD}-\mathrm{WISE}=$ San Diego Wisdom Scale (higher scores $=$ greater wisdom). Gf-EF = executive functioning composite measure of fluid intelligence, presented in sample-based z-score units (higher scores $=$ better performance). Gf-PS = processing speed composite measure of fluid intelligence, presented in z-score units normalized against healthy young adults (higher scores $=$ slower reaction times or worse performance). Episodic memory is presented as a samplebased $\mathrm{z}$-score composite measure (higher scores $=$ better performance)

${ }^{\mathrm{a}} \mathrm{N}=140 .{ }^{\mathrm{b}} \mathrm{N}=137 .{ }^{\mathrm{c}} \mathrm{N}=99$.

composite scores ( $\mathrm{r}=0.181 \quad[95 \%$ CI 0.016, 0.336 ], $\mathrm{p}=.031$; see Figure 1$)$. In other words, approximately $3.28 \%$ of the variance in wisdom was accounted for by Gf-EF. Exploratory multiple regression analyses indicated that the relationship between wisdom and Gf-EF was reduced to nonsignificance upon controlling for age, sex, and education $(\beta=0.114, \mathrm{p}=.214)$.

Wisdom and Gf-PS were not significantly related in zero-order bivariate correlations $(r=0.019$ [95\% CI $-0.179,0.216], p=.854$; see Figure 2 ), sharing only $0.04 \%$ of the total variance with one another. Wisdom and Gf-PS continued to be unrelated in demographically adjusted multiple regression analyses $(\beta=0.094, p=.375)$.

\section{Episodic memory and wisdom}

Wisdom was not significantly related to episodic memory composite scores $(\mathrm{r}=0.109$ [95\% CI $-0.057,0.269], p=.200)$. This relationship remained nonsignificant upon controlling for age, sex, and education in multiple regression analyses $(\beta=0.086, p=.321)$.

\section{Age and wisdom}

Age and wisdom demonstrated a small yet statistically significant negative linear correlation $(\mathrm{r}=-0.197$ [95\% CI $-0.351,-0.033], p=.019$; see Figure 3), which remained significant upon adjusting for sex and education $(\beta=-0.171, p=.049)$. There were no quadratic associations between age and wisdom 


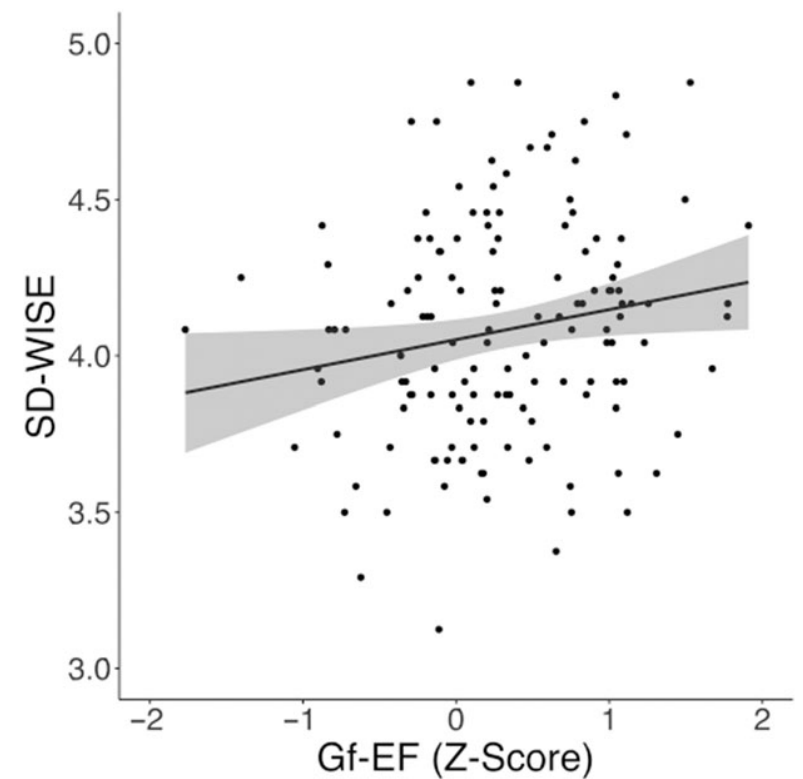

Figure 1. The relationship between executive functioning and wisdom.

The executive functioning composite measure of fluid intelligence (Gf-EF) demonstrated a positive and small yet statistically significant association with wisdom, as assessed by the San Diego Wisdom Scale (SD-WISE). Gf-EF is plotted on the $x$-axis in sample-based z-score units (higher scores = better performance), and SD-WISE is plotted on the $y$-axis (higher scores=greater wisdom). A fitted regression line with $95 \%$ confidence intervals is displayed to help visualize the association.

$(\beta=0.129, p=.907)$, including when adjusting for sex and education $(\beta=0.219, p=.845)$.

\section{Brain structure and wisdom}

dIPFC volumes were not significantly associated with wisdom in multiple regression analyses adjusted for TIV $(\beta=0.033, p=.827)$. dlPFC volumes and wisdom remained unassociated when additionally adjusting for age, sex, and education $(\beta=0.096$, $\mathrm{p}=.538$ ). Similarly, vmPFC volumes were not significantly associated with wisdom $(\beta=-0.010, p=$ $.943)$, including when controlling for demographics $(\beta=0.023, \mathrm{p}=.875)$.

The exploratory, whole-brain VBM analysis did not yield any significant associations between brain structure and wisdom, regardless of whether demographic variables were included within the model.

\section{Discussion}

We empirically tested the magnitude of the relationship between wisdom and Gf using a preregistered analytic plan in a well-characterized cohort of healthy older adults without markers of neurodegenerative disease. The primary findings were

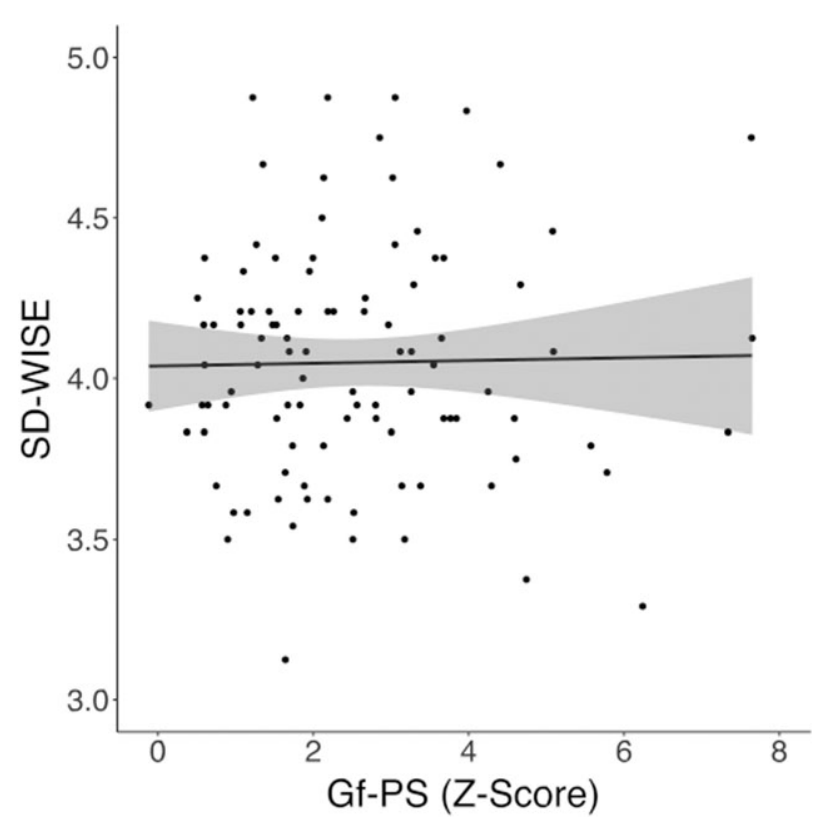

Figure 2. The relationship between processing speed and wisdom. The processing speed composite measure of fluid intelligence (GfPS) was not significantly associated with wisdom, as assessed by the San Diego Wisdom Scale (SD-WISE). Gf-PS is plotted on the $x$ axis in z-score units normalized against healthy young adults (higher scores = slower performance), and SD-WISE is plotted on the $y$-axis (higher scores = greater wisdom). A fitted regression line with $95 \%$ confidence intervals is displayed to help visualize the lack of association.

twofold. First, wisdom demonstrated a small, positive association with Gf-EF that was reduced to nonsignificance upon controlling for age, sex, and education. Second, wisdom and Gf-PS were not significantly related, regardless of demographic covariates. Given that over $95 \%$ of the variance in wisdom was unaccounted for by Gf-EF and GfPS, the present findings do not support an "interrelated" view of wisdom and Gf as largely overlapping constructs. This conclusion is bolstered by the fact that we were adequately powered to detect relationships between wisdom and both Gf-EF (power = 0.95 ) and Gf-PS (power $=0.86$ ), if present, of at least medium effect size.

Our findings are consistent with conceptualizations of wisdom as a multidimensional trait that is far broader than Gf, presumably due to its involvement of a host of social and emotional processes, such as empathy, compassion, affect regulation, and selfreflection, in addition to cognitive skills (Jeste and Lee, 2019). Beyond the obvious theoretical implications of these findings, the observation that wisdom is largely distinct from Gf carries important practical implications. Perhaps most notably, Gf is the strongest cognitive predictor of everyday functioning in older adults (Cahn-Weiner et al., 2007) and consistently declines in later life, with the average 


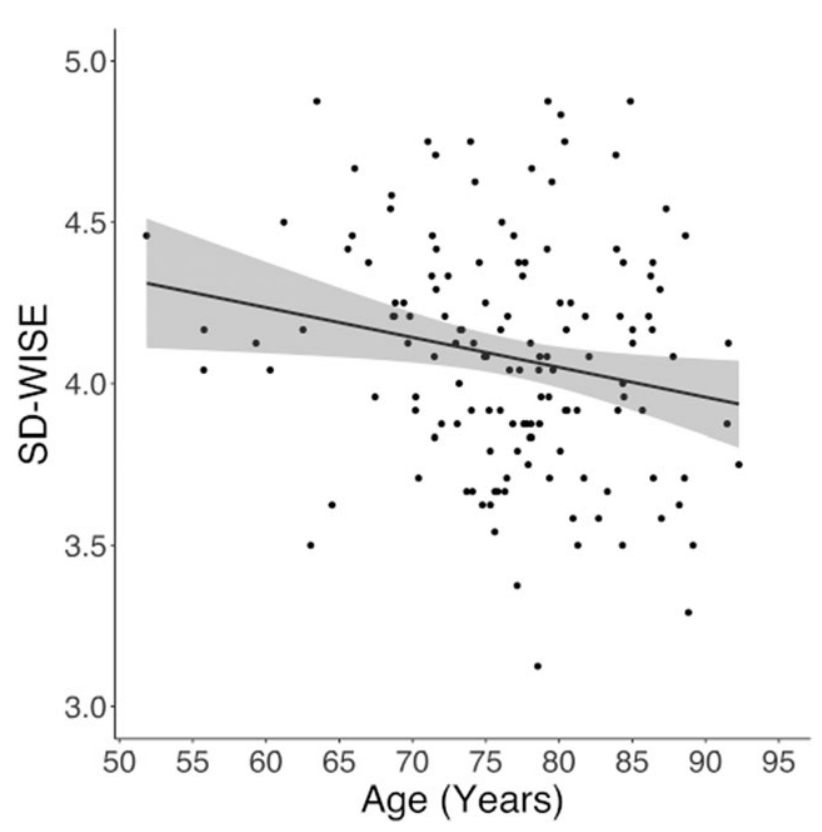

Figure 3. The relationship between age and wisdom. Age demonstrated a negative and small yet statistically significant association with wisdom, as assessed by the San Diego Wisdom Scale (SD-WISE). Age is plotted on the $x$-axis, and SD-WISE is plotted on the $y$-axis (higher scores=greater wisdom). A fitted regression line with $95 \%$ confidence intervals is displayed to help visualize the relationship.

80-year-old performing over 1.5 standard deviations below the average 20-year-old on Gf measures of executive functioning and processing speed (Salthouse, 2004). Yet despite these well-documented declines in Gf, many healthy older adults do not show significant declines in everyday functioning, suggesting a mismatch between cognitive trajectories and functional trajectories. Wisdom may be one critical factor that helps to explain this cognitivefunctional mismatch by bolstering decision-making, interpersonal functioning, and ultimately performance in various daily activities. Consistent with this idea, it has previously been demonstrated that wisdom buffers against functional decline in people with schizophrenia (Van Patten et al., 2019). Future research is warranted to empirically evaluate whether wisdom is similarly protective in older adult populations. Investigating the relationship between wisdom and more "crystallized" aspects of intelligence (Gc), such as the breadth and depth of one's general knowledge base about the world, may also be of interest (McGrew, 2009). For example, it is possible that wisdom helps support overall intellectual, cognitive, and everyday functioning in later stages of life by continuously facilitating the acquisition and application of relevant information to inform behavior and achieve goals (Jeste et al., 2020).
In secondary, exploratory analyses we tested the relationship between wisdom and episodic memory, given the centrality of this cognitive function to aging and many age-associated neurodegenerative disorders (Gorbach et al., 2017). Episodic memory performance was not significantly associated with wisdom. This suggests that, at least in cognitively normal older adults, wisdom may be dissociable from both Gf and non-Gf cognitive functions.

Age and wisdom demonstrated a small, negative correlation, which remained significant upon adjusting for sex and educational attainment. Although wisdom is popularly thought to increase in later life, this has not been consistently supported in the empirical literature, with several prior studies demonstrating weakly negative associations (Mickler and Staudinger, 2008; Thomas et al., 2019) or no associations (Smith and Baltes, 1990; Webster, 2007). More recent work has suggested a curvilinear relationship between age and wisdom across the life span, whereby wisdom peaks in the fifth or sixth decade and then slowly declines thereafter (Ardelt et al., 2018; Thomas et al., 2017; Webster et al., 2014). These discrepant findings likely reflect widespread variability in how wisdom is measured across studies, including which specific subcomponents are assessed (e.g. cognitive, social, and/or emotional), as well as in the age range of the subjects included in a study. In addition, age-related neurodegenerative diseases have been inconsistently ruled out in prior work, which may explain why some studies have suggested a precipitous decline in wisdom among the oldest-old in particular, when risk of dementia is the highest (Staudinger, 1999). Our findings suggest that if wisdom does decline in healthy older adults, the rate of decline is gradual and likely much slower than that of Gf, even among the oldest-old. However, conclusions are limited by the cross-sectional design of the present study.

Although vmPFC and dlPFC have been proposed as two key neuroanatomical substrates of wisdom (Meeks and Jeste, 2009), we did not find any significant associations in the current study. Our null findings do not imply that vmPFC and dlPFC are unimportant for wisdom, but rather that normal variations in gray matter volumes within these regions may be less determinant of wisdom levels among neurologically healthy older adults. It remains possible that a relationship would emerge in patient populations with more significant brain structural changes, such as individuals with neurological injury or disease preferentially impacting prefrontal cortex. For example, patients with behavioral variant frontotemporal dementia, stemming from underlying frontotemporal lobar degeneration, show symptoms that are diametrically opposed to wisdom, such as social inappropriateness, 
impulsivity, apathy, loss of empathy, and lack of insight (Rascovsky et al., 2011).

Beyond our a priori prefrontal brain ROIs, we also performed an exploratory whole-brain VBM analysis to more comprehensively evaluate potential neuroanatomical underpinnings of wisdom, given the lack of prior empirical research on this topic. As with the ROI analyses, the VBM analysis did not yield significant associations. Although we report the VBM findings here in the interest of publishing null results, we acknowledge that we may have been underpowered to detect an effect due to the stringent correction for multiple comparisons that this type of an analysis requires (to avoid inflated Type 1 error rates). Future studies using larger sample sizes will be necessary before drawing any definitive conclusions. In addition, future research may benefit from evaluating relationships between wisdom and more sensitive markers of brain structure and function, such as white matter microstructural integrity or functional connectivity changes, which can precede frank loss of gray matter volume (Sheline and Raichle, 2013). Given the complex, multidimensional nature of wisdom, it is possible that it is subserved by large-scale brain networks working in concert, rather than individual regions.

There are other limitations to the present study that should be considered. Although our findings indicate a dissociation between wisdom and Gf, the cross-sectional design hinders conclusions about how wisdom and Gf may relate to one another over time during the aging process; longitudinal studies are needed to this end. In addition, our sample was predominantly Caucasian (over 90\%) and highly educated (greater than a college degree, on average). Future research is needed in samples that are more diverse while evaluating the potential influence of demographic and cultural factors on expressions of wisdom and its correlates. Finally, it should be acknowledged that wisdom, similar to other personality traits, was assessed by a validated but self-report-based measure, which can be subject to inaccuracies stemming from lack of insight, social desirability, and other forms of response bias (Rosenman et al., 2011), whereas Gf was assessed by performance on objective tests. This difference in administration modality (subjective report versus objective test performance) could have influenced our findings, and future studies may benefit from using performance-based measures of wisdom. That said, cross-modality comparisons are standard practice in the aging literature, particularly when studying personality traits such as wisdom, and it is encouraging that the SD-WISE is a carefully constructed scale with strong theoretical and empirical bases, as well as robust psychometric properties (Thomas et al., 2019). Furthermore, social desirability has not been found to be a significant contributor to SD-WISE scores.

Despite its limitations, the present study is the first to empirically test the magnitude of the relationship between wisdom and Gf in a well-powered study of older adults characterized as neurologically normal. The observation that over $95 \%$ of the variance in wisdom is unaccounted for by measures of Gf helps to delineate and define the construct of wisdom, including its relationship to intelligence, which has been a topic of theoretical debate for decades (Jeste et al., 2020). A better understanding of the wisdom construct also carries important practical implications. Indeed, a growing literature suggests that wisdom plays a central role in successful aging (Lee, 2019) and is associated with a wide range of physical and mental health outcomes in older adults, as well as an overall sense of mastery and purpose in life (Ardelt and Ferrari, 2019). Emerging research further suggests that wisdom promotes healthy social relationships (Auer-Spath and Glück, 2019) and protects against loneliness across the life span, the latter of which is a risk factor for cognitive decline, mood disorders, and mortality (Lee et al., 2019). Given our findings that wisdom appears to be relatively independent from cognitive functions that reliably decline in aging and ageassociated neurodegenerative diseases, wisdom may hold unique potential as a target for interventions to bolster decision-making, social relationships, everyday functioning, and overall health in older adult populations.

A rapidly growing literature provides exciting support for the amenability of various aspects of wisdom to intervention. For example, a metaanalysis of randomized controlled trials (RCTs) showed that nearly half of the psychosocial/behavioral interventions improved components of wisdom such as emotional regulation, empathy, and compassion with medium-to-large effect sizes (Lee et al., 2020). Intervention programs in healthy older adults have also been found to significantly increase overall "emotional intelligence," which encompasses various wisdom-related skills such as emotion regulation and awareness (Delhom et al., 2020). A recent RCT of a group intervention labeled "Raise Your Resilience" improved not only resilience and perceived stress but also overall wisdom, using SDWISE in 89 older residents of five senior housing communities (Treichler et al., 2020). Interestingly, artificial technology has recently been proposed as a novel means of bolstering wisdom in humans, particularly among vulnerable older adult populations with cognitive or psychiatric disorders, and represents an exciting avenue for future research (Jeste et al., 2020).

Taken together, it has become increasingly clear that wisdom holds relevance to numerous health 
outcomes in older adults, and an expanding literature supports its promising potential as a novel target for intervention. However, the success of wisdompromoting interventions will likely depend, at least in part, on a thorough understanding of exactly what this complex, multidimensional trait is and how it relates to other abilities that are highly relevant to the aging process, such as Gf. The present study builds upon prior literature and provides an important step in this direction.

\section{Conflict of interest}

Dr. Staffaroni is funded by the Larry L. Hillblom Foundation (2018-A-025-FEL) and the National Institutes of Health-National Institute on Aging (K23AG061253). Dr. Kramer contributed to the development of the Delis-Kaplan Executive Function System and receives royalties from Pearson Education, Inc., for contributing to the development of the California Verbal Learning Test, is funded by the Larry L. Hillblom Foundation (2018-A-006NET), and is funded by the National Institutes of Health-National Institute on Aging (UCSF ADRC P30AG062422, R01AG032289, and R01AG048234). Dr. Chiong is funded by the National Institutes of Health-National Institute on Aging (R01AG058817 and R01AG022983). None of the aforementioned sponsors played any role in the formulation of the research question, choice of the study design, data collection, data analysis, interpretation of the results, preparation of the manuscript, or the decision to publish. No other study authors have any potential conflicts of interest or disclosures to report.

\section{Description of author(s)' roles}

Dr. Lindbergh assumed a leading role in formulating the research questions, designing the study, analyzing and interpreting the data, and writing the manuscript. Ms. Romero-Kornblum assisted with collecting the data, analyzing the data, and editing the manuscript. Ms. Weiner-Light was involved in data collection, data management, and editing the manuscript. Mr. Young contributed to data collection, data management, visualization of the results, and editing the manuscript. Ms. Fonseca assisted with data collection, data management, and editing the manuscript. Ms. You contributed to study coordination, data collection, data management, and editing the manuscript. Ms. Wolf was involved in data collection, data management, and editing the manuscript. Dr. Staffaroni contributed to data analysis and editing the manuscript. Ms. Daly assisted with data collection and editing the manuscript. Dr. Jeste was involved in designing the study, interpreting the results, and editing the manuscript. Dr. Kramer contributed to study design, formulating the research questions, supervising data collection, interpreting the results, and editing the manuscript. Dr. Chiong was involved in formulating the research questions, designing the study, supervising data collection, interpreting the results, and editing the manuscript.

\section{Acknowledgments}

We would like to acknowledge the research coordinators from UCSF who assisted with data collection and management. We are also very thankful to the Hillblom Aging Network study volunteers and their families who made this work possible. Finally, we would like to acknowledge that our VBM analyses were performed in the Brainsight system, developed at UCSF by Katherine P. Rankin, Cosmo Mielke, and Paul Sukhanov, and powered by the VLSM script written by Stephen M. Wilson, with funding from the Rainwater Charitable Foundation and the UCSF Chancellor's Fund for Precision Medicine.

\section{References}

Ardelt, M. (2003). Empirical assessment of a threedimensional wisdom scale. Research on Aging, 25, 275-324. https://doi.org/10.1177/0164027503025003004

Ardelt, M. and Ferrari, M. (2019). Effects of wisdom and religiosity on subjective well-being in old age and young adulthood: exploring the pathways through mastery and purpose in life. International Psychogeriatrics, 31, 477-489. https://doi.org/10.1017/S1041610218001680

Ardelt, M., Pridgen, S. and Nutter-Pridgen, K. L. (2018). The relation between age and three-dimensional wisdom: variations by wisdom dimensions and education. The Fournals of Gerontology: Series B, 73, 1339-1349.

Ashburner, J. (2007). A fast diffeomorphic image registration algorithm. NeuroImage, 38, 95-113. https://doi .org/10.1016/J.NEUROIMAGE.2007.07.007

Auer-Spath, I. and Gluck, J. (2019). Respect, attentiveness, and growth: wisdom and beliefs about good relationships. International Psychogeriatrics, 31, 1809 1821. https://doi.org/10.1017/S104161021900022X

Baltes, P. and Smith, J. (1990). Toward a psychology of wisdom and its ontogenesis. In: R. J. Sternberg (Ed.), Wisdom: Its Nature, Origins, and Development (pp. 87-120). New York, NY: Cambridge University Press.

Bangen, K. J., Meeks, T. W. and Jeste, D. V. (2013). Defining and assessing wisdom: a review of the literature. American fournal of Geriatric Psychiatry, 21, 1254-1266. https://doi.org/10.1016/j.jagp.2012.11.020

Cahn-Weiner, D. A. et al. (2007). Cognitive and neuroimaging predictors of instrumental activities of daily living. Fournal of the International Neuropsychological Society, 13, 747-757. https://doi.org/10.1017/S1355617707070853 
Cattell, R. B. (1943). The measurement of adult intelligence. Psychological Bulletin, 40, 153-193. https://doi.org/10.1037/ h0059973

Delgado, M. R. et al. (2016). Viewpoints: dialogues on the functional role of the ventromedial prefrontal cortex. Nature Neuroscience, 19, 1545-1552. https://doi.org/10.1038/nn .4438

Delhom, I., Satorres, E. and Melendez, J. C. (2020). Emotional intelligence intervention in older adults to improve adaptation and reduce negative mood. International Psychogeriatrics. https://doi.org/10.1017/ S1041610220003579

Delis, D., Kaplan, E. and Kramer, J. (2001). Delis-Kaplan Executive Function System. San Antonio, TX: Psychological Corporation.

Delis, D., Kramer, J., Kaplan, E. and Ober, B. (2000). California Verbal Learning Test-Second Edition. San Antonio, TX: Psychological Corporation.

Desikan, R. S. et al. (2006). An automated labeling system for subdividing the human cerebral cortex on MRI scans into gyral based regions of interest. NeuroImage, 31, 968 980. https://doi.org/10.1016/J.NEUROIMAGE.2006.01 .021

Diamond, A. (2013). Executive functions. Annual Review of Psychology, 64, 135-168. https://doi.org/10.1146/annurevpsych-113011-143750

Friston, K., Ashburner, J., Kiebel, S., Nichols, T. and Penny, W. (2011). Statistical Parametric Mapping: The analysis of functional brain images. London, UK: Academic Press.

Gorbach, T. et al. (2017). Longitudinal association between hippocampus atrophy and episodic-memory decline. Neurobiology of Aging, 51, 167-176. https://doi.org/10 .1016/j.neurobiolaging.2016.12.002

Grossmann, I., Na, J., Varnum, M. E. W., Park, D. C., Kitayama, S. and Nisbett, R. E. (2010). Reasoning about social conflicts improves into old age. Proceedings of the National Academy of Sciences of the United States of America, 107, 7246-7250. https://doi.org/10.1073/pnas .1001715107

Hale, S. and Myerson, J. (1996). Experimental evidence for differential slowing in the lexical and nonlexical domains. Aging, Neuropsychology, and Cognition, 3, 154-165. https:// doi.org/10.1080/13825589608256621

Happé, F. G., Winner, E. and Brownell, H. (1998). The getting of wisdom: Theory of mind in old age. Developmental Psychology, 34, 358-362. https://doi.org/10.1037//00121649.34.2.358

Jeste, D. V., Ardelt, M., Blazer, D., Kraemer, H. C., Vaillant, G. and Meeks, T. W. (2010). Expert consensus on characteristics of wisdom: A Delphi method study. Gerontologist, 50, 668-680. https:/doi.org/10.1093/geront/ gnq022

Jeste, D. V., Graham, S. A., Nguyen, T. T., Depp, C. A., Lee, E. E. and Kim, H. (2020). Beyond artificial intelligence: exploring artificial wisdom. International Psychogeriatrics, 32, 993-1001. https://doi.org/10.1017/ S1041610220000927

Jeste, D. V. and Lee, E. E. (2019). The emerging empirical science of wisdom. Harvard Review of Psychiatry, 27, 127140. https://doi.org/10.1097/HRP.0000000000000205
Jeste, D. V. and Vahia, I. V. (2008). Comparison of the conceptualization of wisdom in ancient Indian literature with modern views: focus on the Bhagavad Gita. Psychiatry, 71, 197-209. https://doi.org/10.1521/psyc.2008.71.3.197

Jeste, D. V et al. (2019). Study of independent living residents of a continuing care senior housing community: sociodemographic and clinical associations of cognitive, physical, and mental health. The American fournal of Geriatric Psychiatry, 27, 895-907. https://doi.org/10.1016/j .jagp.2019.04.002

Jonaitis, E. M. et al. (2019). Measuring longitudinal cognition: individual tests versus composites. Alzheimer's and Dementia: Diagnosis, Assessment and Disease Monitoring, 11, 74-84. https://doi.org/10.1016/j.dadm.2018.11.006

Jung, R. E. and Haier, R. J. (2007). The Parieto-Frontal Integration Theory (P-FIT) of intelligence: converging neuroimaging evidence. Behavioral and Brain Sciences, 30, 135-154. https://doi.org/10.1017/S0140525X07001185

Kerchner, G. A. et al. (2012). Cognitive processing speed in older adults: relationship with white matter integrity. PLoS ONE, 7, 1-10. https://doi.org/10.1371/journal.pone .0050425

Kievit, R. A., Davis, S. W., Griffiths, J., Correia, M. M., Cam-CAN and Henson, R. N. (2016). A watershed model of individual differences in fluid intelligence. Neuropsychologia, 91, 186-198. https://doi.org/10.1016/j .neuropsychologia.2016.08.008

Kramer, J. H. et al. (2003). Distinctive neuropsychological patterns in frontotemporal dementia, semantic dementia, and Alzheimer disease. Cognitive and Behavioral Neurology, $16,211-218$.

Lee, E. E. (2019). Aging successfully and healthfully. International Psychogeriatrics, 31, 439-441. https://doi.org/10. 1017/S1041610219000012

Lee, E. E. et al. (2019). High prevalence and adverse health effects of loneliness in community-dwelling adults across the lifespan: role of wisdom as a protective factor. International Psychogeriatrics, 31, 1447-1462. https://doi.org/10.1017/ S1041610218002120

Lee, E. E. et al. (2020). Outcomes of randomized clinical trials to enhance social, emotional, and spiritual components of wisdom: a systematic review and metaanalysis. $\mathcal{F A M A}$ Psychiatry, 77, 925-935. https://doi.org/10. 1001/jamapsychiatry.2020.0821

Lindbergh, C. A. et al. (2019). Systemic tumor necrosis factor-alpha trajectories relate to brain health in typically aging older adults. The fournals of Gerontology: Series A. https://doi.org/10.1093/gerona/glz209

Luna, B., Padmanabhan, A. and O'Hearn, K. (2010). What has fMRI told us about the Development of Cognitive Control through Adolescence? Brain and Cognition, 72, 101-113. https://doi.org/10.1016/j.bandc.2009.08.005

Mazziotta, J. C., Toga, A. W., Evans, A., Fox, P. and Lancaster, J. (1995). A probabilistic atlas of the human brain: theory and rationale for its development. NeuroImage, 2, 89-101. https://doi.org/10.1006/nimg.1995. 1012

McGrew, K. S. (2009). CHC theory and the human cognitive abilities project: standing on the shoulders of the giants of psychometric intelligence research. Intelligence, 37, 1-10. https://doi.org/10.1016/j.intell.2008.08.004 
Meeks, T. W. and Jeste, D. V. (2009). Neurobiology of wisdom: a literature overview. Archives of General Psychiatry, 66, 355-365. https://doi.org/10.1001/archgenpsychiatry .2009 .8

Mickler, C. and Staudinger, U. M. (2008). Personal wisdom: validation and age-related differences of a performance measure. Psychology and Aging, 23, 787-799. https://doi.org/10.1037/a0013928

Rascovsky, K. et al. (2011). Sensitivity of revised diagnostic criteria for the behavioural variant of frontotemporal dementia. Brain, 134, 2456-2477. https://doi.org/10 $.1093 /$ brain/awr 179

Rosenman, R., Tennekoon, V. and Hill, L. G. (2011). Measuring bias in self-reported data. International fournal of Behavioural and Healthcare Research, 2, 320-332. https:// doi.org/10.1504/ijbhr.2011.043414

Sallet, J. et al. (2013). The organization of dorsal frontal cortex in humans and macaques. Fournal of Neuroscience, 33, 12255-12274. https://doi.org/10.1523/JNEUROSCI $.5108-12.2013$

Salthouse, T. A. (2004). What and when of cognitive aging. Current Directions in Psychological Science, 13, 140-144. https://doi.org/10.1111/j.0963-7214.2004.00293.x

Sheline, Y. I. and Raichle, M. E. (2013). Resting state functional connectivity in preclinical Alzheimer's disease. Biological Psychiatry, 74, 340-347. https://doi.org/10.1016/ j.biopsych.2012.11.028

Sheppard, L. D. and Vernon, P. A. (2008). Intelligence and speed of information-processing: a review of 50 years of research. Personality and Individual Differences, 44, 535-551. https://doi.org/10.1016/j.paid.2007.09.015

Sled, J. G., Zijdenbos, A. P. and Evans, A. C. (1998). A nonparametric method for automatic correction of intensity nonuniformity in MRI data. IEEE Transactions on Medical Imaging, 17, 87-97. https://doi.org/10.1109/42.668698

Smith, J. and Baltes, P. B. (1990). Wisdom-related knowledge: age/cohort differences in response to lifeplanning problems. Developmental Psychology, 26, 494505. https://doi.org/10.1037/0012-1649.26.3.494

Sperling, R. A. et al. (2011). Toward defining the preclinical stages of Alzheimer's disease: recommendations from the National Institute on Aging-Alzheimer's Association workgroups on diagnostic guidelines for Alzheimer's disease. Alzheimer's and Dementia, 7, 280-292. https://doi .org/10.1016/j.jalz.2011.03.003

Staffaroni, A. M. et al. (2018). The longitudinal trajectory of default mode network connectivity in healthy older adults varies as a function of age and is associated with changes in episodic memory and processing speed. The fournal of Neuroscience, 38, 2809-2817. https://doi.org/10.1523/ JNEUROSCI.3067-17.2018
Staudinger, U. M. (1999). Older and wiser? Integrating results on the relationship between age and wisdom-related performance. International fournal of Behavioral Development, 23, 641-664. https://doi.org/10.1080/ 016502599383739

Sternberg, R. J. (1985). Implicit theories of intelligence, creativity, and wisdom. Fournal of Personality and Social Psychology, 49, 607-627. https://doi.org/10.1037/00223514.49.3.607

Sternberg, R. J. (2005). Older but not wiser? The relationship between age and wisdom. Ageing International, 30, 5-26. https://doi.org/10.1007/BF02681005

Thomas, M. L., Bangen, K. J., Ardelt, M. and Jeste, D. V. (2017). Development of a 12-Item Abbreviated ThreeDimensional Wisdom Scale (3D-WS-12): item selection and psychometric properties. Assessment, 24, 71-82. https:// doi.org/10.1177/1073191115595714

Thomas, M. L. et al. (2019). A new scale for assessing wisdom based on common domains and a neurobiological model: The San Diego Wisdom Scale (SD-WISE). Fournal of Psychiatric Research, 108, 40-47. https://doi.org/10.1016/J .JPSYCHIRES.2017.09.005

Treichler, E. B. H. et al. (2020) A pragmatic trial of a group intervention in senior housing communities to increase resilience. International Psychogeriatrics, 32, 173-182. doi .org/10.1017/S1041610219002096

Van Aken, L., Kessels, R. P. C., Wingbermühle, E., Van Der Veld, W. M. and Egger, J. I. M. (2015). Fluid intelligence and executive functioning more alike than different? Acta Neuropsychiatrica, 28, 31-37. https://doi.org/ 10.1017/neu.2015.46

Van Patten, R., Lee, E. E., Daly, R., Twamley, E., Tu, X. M. and Jeste, D. V. (2019). Assessment of 3dimensional wisdom in schizophrenia: associations with neuropsychological functions and physical and mental health. Schizophrenia Research, 208, 360-369. https://doi .org/10.1016/j.schres.2019.01.022

Webster, J. (2007). Measuring the character strength of wisdom. International fournal of Aging and Human Development, 65, 163-183. https://doi.org/10.2190/AG.65 .2.d

Webster, J. D., Westerhof, G. J. and Bohlmeijer, E. T. (2014). Wisdom and mental health across the lifespan. The fournals of Gerontology Series B: Psychological Sciences and Social Sciences, 69, 209-218. https://doi.org/10.1093/geronb/ gbs 121

Worthy, D. A., Gorlick, M. A., Pacheco, J. L., Schnyer, D. M. and Maddox, W. T. (2011). With age comes wisdom: decision making in younger and older adults. Psychological Science, 22, 1375-1380. https://doi.org/10. $1177 / 0956797611420301$ 